\title{
Evaluation of Video Transmission in Emergency Response Ad Hoc Networks
}

\author{
C. Bouras ${ }^{1,2}$, G. Gioumourtzis ${ }^{3}$, A. Gkamas ${ }^{4}$, V. Kapoulas ${ }^{1}$, D. Politaki ${ }^{2}$, and E. Tsanai ${ }^{2}$

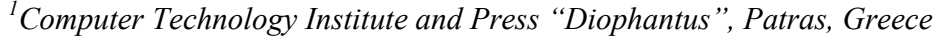 \\ ${ }^{2}$ Computer Engineering and Informatics Dept., University of Patras, Patras, Greece \\ ${ }^{3}$ Center for Security Studies, Athens, Greece \\ ${ }^{4}$ University Ecclesiastical Academy of Vella, Ioannina, Greece \\ \{bouras,kapoulas\}@cti.gr,kioumourtzis@gmail.com,gkamas@aeavellas.gr,\{politaki,tsanai\}@ceid.upatras.gr
}

Keywords: $\quad$ MANETs, Multi-interfaces and Multi-channels, Video Transmission, Emergency Response.

\begin{abstract}
Mobile Ad hoc NETworks (MANETs) are becoming more essential to wireless communications due to growing popularity of mobile devices. MANETs are also essential in Emergency Response situation where network information flow between deployed units is vital. In such cases voice and video transmission is essential to form a global view of the situation and decide on action. However, MANETs do not seem to effectively support multimedia applications and especially video transmission. In this work we evaluate the use of multiple interfaces and multiple channels for Video Transmission in Emergency Response Ad hoc Networks. We also evaluate the video transmission with rate adaptation. Aim of this paper is to tune multiinterfaces and multi-channels video transmission mechanisms in order to support better emerging situations and offer a solution to have acceptable transmission of the necessary video streams so that the control can get a complete overall picture of the situation and decide on the action to be taken. The simulation evaluation performed shows that multi-interfaces and multi-channels video transmission offers many advantages in Video Transmission in Emergency Response Ad hoc Networks.
\end{abstract}

\section{INTRODUCTION}

Recent disasters scaled from big catastrophes (Hurricane Katrina USA 2005, Tsunami Japan, 2011, Earthquake Japan 2011) to smaller earthquakes (City of Patras, Greece, 2011) resulted in the collapse of telecommunication networks. Under these circumstances, one way to set up a communication network, which can provide a variety of applications, is the deployment of Mobile Ad hoc Networks (MANETs).

MANETs are decentralized, self-organized, and capable of restoring communications without depending on any infrastructure. Each node is equipped with wireless modules that enable the communication with any other node in the network over the wireless channel. All nodes can act, if necessary, as intermediate nodes for routing data packets to their final destination. In other words, MANETs allow data transmission between nodes that are out of range, through multiple hops.

MANETs have significant advantages over traditional telecommunication networks. They are cheaper, because they do not require any infrastructure and more robust, because of nonhierarchical structure and network management mechanisms. The most important attributes of MANETs are mobility and flexibility as they can be organized and dispersed in very short time.

The latest advances in MANETs, disclosed a number of new proposals in an effort to cover almost all the open issues. New routing protocols based on the Global Positioning Services (GPS) provide even more efficient routing than earlier approaches. Quality of Service (QoS) is enabled with new application-aware MAC protocols (IEEE 802.11e). Cross-layer design and adaptive video coding try to increase Quality of Experience (QoE) for the end users.

The implementation on MANETs on real-life applications seems to be very limited. The new generation of cellular networks that offer higher bit rates, Internet connectivity, smart devices, and wider coverage make them very attractive for satisfying the implementation of the aforementioned applications. In contrast, MANETs are still facing a 
number of open issues that are under consideration by the research community. These include efficient routing, quality of service (QoS), energy consumption, and security.

One application area of MANETs being studied the last few years is disaster management (Jiazi, 2008), (Goyal et al., 2011).

Other solutions have been presented in order to increase MANETs applicability in crisis related scenarios. As far as routing is concerned, new adaptive protocols have been proposed that rely on the three standard routing protocols, namely Ad hoc On-Demand Distance Vector (AODV) (Perkins et al., 2003), Dynamic Source Routing Protocol (DSR) (Johnson et al., 2007), and Optimized Link State Routing Protocol (OLSR) (Clausen and Jacquet, 2003). In (Panaousis et al., 2010) emergency eMANETs are presented as the networks consisted mostly from intelligent devices such as smart phones and PDAs, using an adaptive routing protocol called ChaMeLeon (CML). The CML defines the size of the network Critical Area (CA) and based on this information it routes using AODV in small topologies and OLSR in larger. Although this approach tries to exploit the characteristics of reactive routing (AODV), which are suited better in small areas and the reactive ones (OLSR) in large areas, in cases of moving nodes inside and out of the network, the CML is not effective. In (Ko and Vaidya, 2000) the Location Aided-Routing (LAR) improves the AODV's route discovery with the help of GPS coordinates that are used for the estimation of the destination's possible location. In LAR, the source is aware of the destination node's position and speed and based on this information it limits the search area. In contrast, the GPS enhanced routing protocol GeoAODV (Hosseini-Pozveh et al., 2009) which also is a variation of AODV, dynamically learns and distributes location information among the nodes in the network topology. Another version of LAR namely Greedy Location-Aided Routing (GLAR) (Wang et al., 2009) aims in the reduction of the total number of routing packets (RREQ and RREP messages) travelling in the network. Another GPS enhanced routing protocol is the Greedy Perimeter Stateless Routing GPSR (Karp and Kung, 2000) that makes greedy forwarding decisions by keeping information only of the immediate neighbors of the intermediate node. In (Karp and Kung, 2000), GPSR outperformed DRS in successful data packet delivery in high numbers of nodes. In (Dwivedi et al., 2012), a Hybrid Dd hoc Network (HANET) is proposed which is a combination of Static Ad hoc Network (SANET) and Mobile Ad hoc Network (MANET). This mesh network model can be easily built in situations where communications, power and roads get disrupted. This model includes a MAC protocol based on the directional smart antenna and the results have shown better throughput and end-to-end performance than the legacy MANETs with IEEE 802.11-MAC. Another type of solution in (Kumar and Kumar, 2011) is a framework for disaster management. According to this solution, depending on the disaster grade of the alertness (DGA) three phases of management are analyzed (Most Critical Phase, Optimal Power Phase, Average Reliable and Power Phase). The simulations showed that Most Critical Phase is the most reliable model in data transmission with the fewer hops but with the most energy consumption.

Last, but not least, there is a great interest on the use of Multi-Interfaces and Multi-Channels (MIMC) in MANETs. The rapid growth of IEEE 802.11 technology has eased the sharp decrease of multiinterface enabled devices' prices and therefore, their presence is each day more and more common. Several efforts have been made in the last years in order to implement and attach the technology of MIMC on the mobile nodes.

However, it is our feeling that current research and state-of-the-art solutions are not so mature to provide general solutions for efficient implementation of MANETs in all the application areas. What is needed in fact is to apply research results with the existing vendor technology for addressing specific problems in a specific application area. And indeed, it is a common understanding that there is no, for instance, an efficient routing protocol that can be used to any MANET topology, regardless the number of mobile nodes, the traffic type and the QoS constrains for delay sensitive applications.

In this work we evaluate the multi-interfaces and multi-channels Video Transmission in Emergency Response Ad hoc Networks. Target of this paper is to tune multi-interfaces and multi-channels video transmission mechanisms in order to support better emerging situations (taking into account e.g. the number of deployed units, their dispersion, the available equipment, etc.) and offer a solution to have acceptable transmission of the necessary video streams so that the control can get a complete overall picture of the situation and decide on the action to be taken.

The proposed solution makes use of the ability of the nodes to have more than one wireless interface and thus make use of more than one channel for 
simultaneously receiving and transmitting video data. This ability is due to the unique nature that the equipment of each node may have, as they can be built to suit the needs of the emergency response personnel, i.e. they are not commercial of the shelf (COTS) devices).

The proposed solution also makes use of multiple channels. This is also applicable in emergency response situations (e.g. a fire in the woods) as it is expected that in the area there will not be any other devices making use of the available wireless channels.

Therefore, in this work we do not intent to provide a common solution for all application areas and topologies in MANETs. We rather try to integrate existing solutions and/or modify them in order to provide efficient services in a specific application area. We base this work on existing operational knowledge of the Hellenic Fire Brigade that has been obtained in a number of physical disasters across the country, over the last years.

On the basis of end-user participation we endeavor to provide an end-user driven solution.

The rest of the paper is organized as follows. In Section II we discuss related work and in Section III we list specific End-User requirements. In Section IV we present the proposed design for efficient support to Fire Brigade in a disaster scenario, followed by performance evolution in Section V. We conclude our paper in Section VI, with thoughts for future work.

\section{END-USER REQUIREMENTS}

In order to obtain end-user requirements and identify capability gaps an interview was taken place by representatives from the Hellenic Fire Brigade. The interview results are summarized in the following paragraphs.

Currently, one of the main problems of the First Responders (FR) (police, fire brigade, coast guard, port authorities, etc.) in disasters is the lack of availability and the low flow rate of relevant information both at the First Responder and the local manager level.

Loss of communications and positioning, lack of information on the environment (high temperature, hazardous gas, etc.) and low efficiency of the Human Machine Interface (HMI) are the main current issues that demand resolution.

In effect, during the intervention there is a gap between the First Responders' situation (positioning, health, etc.) and the overall overview at their mobile headquarter or at their coordination and operation center, which increases the response time and respectively reduces the available action time.

Four areas were identified for providing technological solutions that will allow for enhanced intervention procedures to be faster, more efficient and safer. In that respect, the solutions foreseen by the end-user are the following:

- Communication: There is a need to enhance the communication among the First Responders on the field, the units and their Head Quarter (HQ) by providing self-organizing, robust ad hoc communications in cases where the existing infrastructure may be compromised.

- Personnel/Vehicle Positioning: The extraction of accurate positioning in outdoor environments is of tremendous importance for better coordination and allocation of resources.

- Area/Threat Identification Sensors: It is of vital importance, in any operation, for the First Responders to be equipped with sensors that offer a reliable overview of the situation and of potential threats (e.g. Chemical, biological, radiological, nuclear, fire, etc.), in order to provide more accurate situation awareness and enhanced decision making.

- Human Machine Interface (HMI): When implementing the aforementioned solutions, First Responders' small time frame must not be ignored. For that reason the HMI plays an important role in the reading, sending and continuity of real-time information.

The situational awareness of first responders is confined in the following application areas (specific end-user requirements):

- Voice

- Video streaming

- Data (reports)

- Imaging

- GIS information for Command and Control

- Smog and toxic Sensors

Our solution aims at reducing any delay in response, increasing the effectiveness and safety of First Responders by means of maximizing information gathering and communication with higher command levels, while simultaneously reducing risk and increasing chances of survival for both the rescued and the personnel involved. 


\section{PROPOSED DESIGN}

\subsection{Description of the Mechanism}

The proposed approach will be based on the concept of usage of multiple channels (Gharavi, 2008) for video transmission. This approach offers higher aggregate bandwidth and fault tolerance and could prevent interference and improve network performance.

The main problem with the bandwidth usage when a video is transmitted over a multi-hop route in the MANET is that every intermediate node cannot transmit when its previous and next nodes in the path are transmitting. Thus even in the simple case of one video transmission only $1 / 3$ of the effective bandwidth is usable.

The situation is even worst when nodes of other paths are in the vicinity and get their share of the bandwidth.

In order to overcome this problem we observe that the areas requiring emergency response are more likely without any existing wireless networks, or the existing wireless network are most probably out of order because of the situation that requires the emergency response (in any case any existing wireless network may be forced to cease operation in order to facilitate the emergency response team's MANET operation).

Therefore the MANET is free to use all the available channels.

We propose that each node carries multiple radios and is able to operate concurrently in different channels. In any routing path, adjacent links use different channels, and therefore it is possible: a) for each node to concurrently communicate with multiple neighbors, and b) that each node can transmit along the routing path, concurrently with its previous and next node.

The above mechanism allows the MANET to utilize the links as if the medium is not shared, thus multiplying its capacity.

\subsection{Reference Scenario}

The reference scenario refers to a post-disaster area in which the current fixed infrastructure has been destroyed. We can visualize the deployment of first responders (e.g., Fire Brigade, or other rescue teams) to provide relief to disaster area. The network topology includes:

- A fixed command Post (operational level)

- Mobile regional command posts (tactical level)
- Several teams deployed in the area (field level)

Fig. 1 depicts this scenario. The fixed command post is required to have connection to external network. This can be realized by satellite links depending on the availability. However, our solution mainly targets at connecting the fixed Command Post with the lower level of commands; tactical and field level. In this case WiMax technology can provide a feasible solution. At the tactical level the Command Post is equipped with devices with dual interfaces. WiMax interface is used to provide connectivity with the operational level whereas Wifi (IEEE $802.11 \mathrm{x})$ to communicate with the mobile teams. Regional Commands communicate with each other with WiMax and they use an Ad hoc routing protocol. Regional Commands, mobile team leaders and rescuers (mobile nodes) use the same routing protocol creating in this way a mess network. There may be cases in which a node in the field may be able to have connectivity with a neighboring regional command.

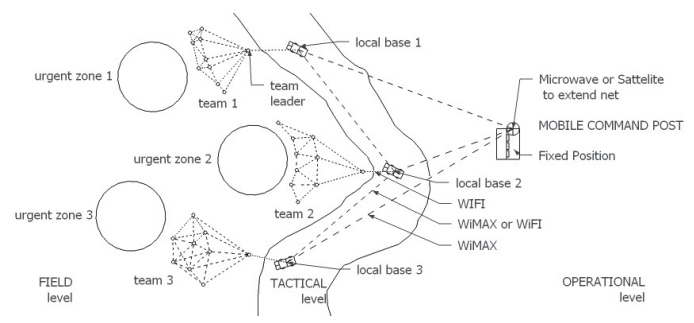

Figure 1: Reference scenario.

\subsection{Selection of Video Coding/Quality}

H.264/MPEG-4 Part 10 or AVC (Advanced Video Coding) is currently one of the most commonly used formats for the recording, compression, and distribution of high definition video over networks. H.264 poised to become the de facto standard for format of convergence in the digital video industry regardless of the video playback platform. The intention behind H.264/AVC project was to provide good video quality at substantially lower bit rates than previous standards. An additional goal was to provide enough flexibility to allow the standard to be applied to a wide variety of applications on a wide variety of networks and systems. H.264/AVC/MPEG-4 Part 10 contains Multi-picture inter-picture prediction including the features like using previously-encoded pictures as references in a more flexible way than in past standards, allowing up to 32 reference pictures to be used sometimes. In addition H.264 is more attractive for video network delivery and for delivery of $\mathrm{HD}$, high definition 
video and is used by many video delivery sites (like YouTube, iTunes etc). Moreover H.264 or AVC is an open format with published specification and is available for anyone to implement.

\subsection{Selection of Transport Protocol}

Multimedia applications have enjoyed the global interest over the last few years. These applications are characterized by three main properties: the demand for high data transmission rate (bandwidthconsuming applications), the sensitiveness to packet delays (latency and jitter) and the tolerance to packet losses (packet-loss tolerant applications).

The Real Time Protocol (RTP) and the associated RTP Control Protocol (RTCP) protocols (Schulzrinne et al., 2003) constitute the de facto standard and is the dominant transport protocol for multimedia data transmission. RTP is a real time transport protocol that is used usually on top of the UDP protocol (also other transport protocols are supported). The main functions of the RTP include: Identification of payload type, identification of the source sending the RTP packets, timestamps to the RTP packets and sequence numbers of the RTP packets.

The RTCP protocol provides to participants of the RTP session feedback information of the network conditions. The main functions of the RTCP are: Network measurements for QoS (packet loss ratio, delay jitter, timestamps of sender and receiver reports etc), identification of the source sending the RTCP packets and estimation of the session size and scaling mechanisms.

\section{PERFORMANCE EVALUATION}

\subsection{NS-2 based Simulations}

As far as NS-2 (Network Simulator) is concerned, TeNs (The Enhanced Network Simulator, http:// www.cse.iitk.ac.in/users/braman/tens/), (Raniwala and Chiueh 2005), and (Agüero Calvo and Pérez Campo, 2007) are the most complete previous works for MIMC technology. An older project, MITF (which was discontinued, and is no longer available) was carried out at the University of Rio de Janeiro and its goal was to adapt MIMC technology to the AODV routing protocol in ns-2.28 (see, Agüero Calvo and Pérez Campo, 2007). TeNs was implemented at the Indian Institute of Technology of Kanpur-India, and its main goal was to add multiinterface support for ns-2.1b9a and improve its implementation of the IEEE 802.11 protocol. The project Hyacinth was conducted at the University of New York for ns-2.29a and could be extended for use at ns-2.29. These three projects add many capabilities concerning the implementation of MIMC in NS-2, but do have many drawbacks. Static configuration, low flexibility in routing protocol and inability to develop various tcl scripts are such drawbacks. However, the model of Agüero Calvo and Pérez Campo (2007) about the MIMC implementation in NS-2 is much more flexible and complete as it is based on the combination of all the previous projects referred above. In (Agüero Calvo and Pérez Campo, 2007) a detailed set of changes that need to be performed on the simulation framework is presented, in order to use a flexible number of interfaces and channels per node.

In our approach, we follow the model of Agüero Calvo and Pérez Campo (2007) to support MIMC technology in our simulations.

The simulations concerns an emergency response situation where a command $\&$ control centre is setup and group or units are dispatched to different areas require assistance. In each area the group of units has a (mobile) local center and units are deployed locally to confront the situation. Each unit is a node in the network. The number of groups is in the order of 5-10 and the number of units in each group is similarly in the order of 5-15. The distance between the deployed units and the local center varies up to half kilometer, while the distance between the command \& control and the group centers varies up to 10 kilometers.

In the initial simulations only one group is simulated, to study the performance of video transmission within the group and towards the local centre.

\subsection{Evaluation Results Analysis}

The simulations are run for two different random placements of the group members in the vicinity of their site, to avoid bias due the group member placement in the results. The results were quite similar, so only the one case is presented below.

In order to account for possibly bad conditions (which are expected in emergency response situations) the maximum data rate (bandwidth) of each wireless connection is set to $1 \mathrm{Mbit} / \mathrm{sec}$.

In each simulation 3 videos are transmitted. Each video is transmitted using variable bit rate (VBR) with a mean rate of $0.32 \mathrm{Mbit} / \mathrm{sec}$. This (when taking into account the protocol overhead) slightly saturates the wireless link at the receiving node and 
demonstrates the bottlenecks. The videos last for just less than a minute.

Figure 2 shows the videos' transmission rates of three simultaneous video transmissions without using multiple interfaces and multiple channels per node. The rate of each video of each video fluctuates a lot. Figure 3 shows that, as mentioned above, the system is saturated and this explain the fluctuation as the three streams compete with each other for bandwidth.

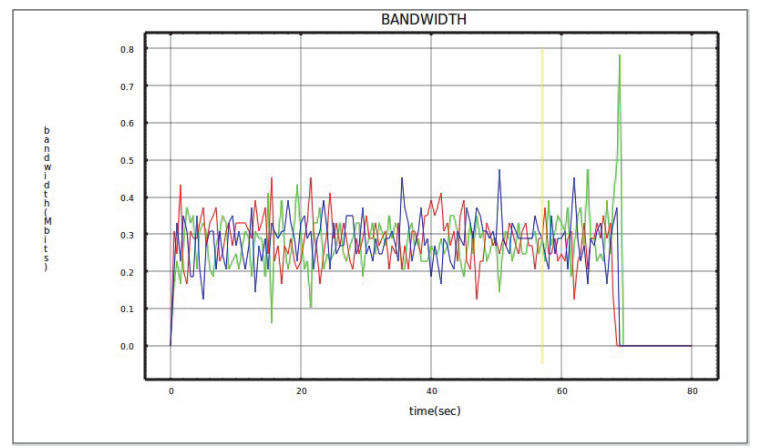

Figure 2: Bandwidth usage for 3 videos transmission without MIMC support.

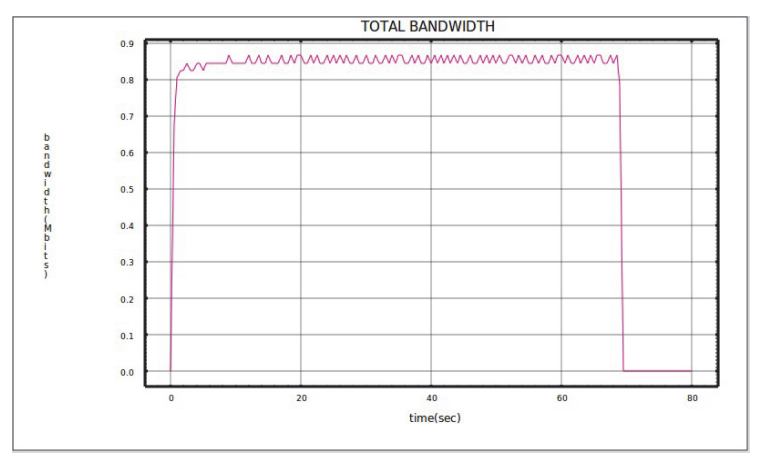

Figure 3: Cumulative bandwidth for 3 videos transmission without MIMC support.

In addition the average bandwidth for each video is slightly less than the mean bit rate and thus the videos arrive with some delay. Figure 4 shows the delay for the three videos and demonstrates how it is accumulated. At the end of the videos this delay is almost $10 \mathrm{sec}$. This means that video transmission is impractical as in a real situation live video is streamed and the delay accumulates, and at some point the centre receives very outdated video.

It should be noted that the situation is similar with only two and even with only one video transmission, in case the video transmits over three or more. Then, when an intermediate node transmits then neither its previous not its next node can transmit at the same time. Thus, only one third of the bandwidth is usable at any time.

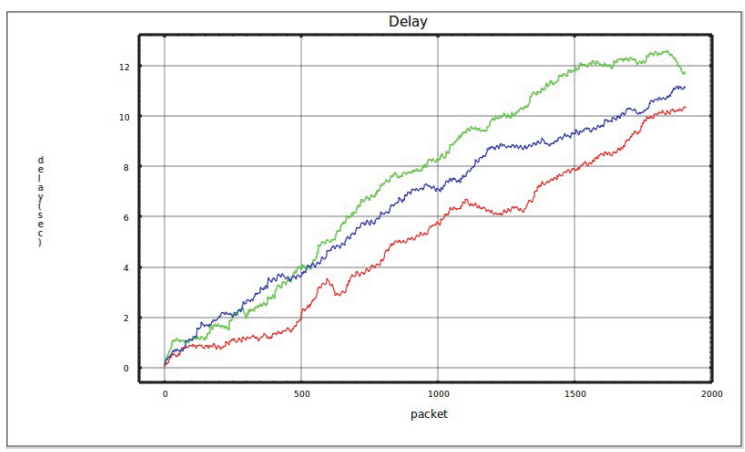

Figure 4: Delay for 3 videos transmission without MIMC support.

It is clear that by using the same channel for all the wireless connections the ad hoc network cannot properly support the transmission of even a few video streams.

In order to utilize multiple channels, however the nodes must be equipped with multiple wireless interfaces (so that each node can receive and transmit at the same time over different channels).

This is not an unreasonable assumption for emergency response teams, as their equipment can be tailor made to their specific requirements.

We repeat the simulations by equipping each node with two wireless interfaces and the ability to use multiple channels. The node for the centre is equipped with three wireless interfaces as it is the sink of the video transmissions and needs to be able to receive over more channels.

Figure 5 shows the bandwidth usage of the three videos in the receiving node. All videos are transmitted at their intended rate (as they arrive at different interfaces over different channels), and there are no fluctuations other than those from the VBR encoding of the videos (which is very important during real time multimedia transmission).

Figure 6 shows the cumulative bandwidth usage. This exceeds the capacity of one wireless link at all times (if we take into account the protocol overhead the). However this is to be expected as the total capacity is now two-fold for all nodes and three-fold for the centre node. Therefore there is available capacity to accommodate more video transmissions.

Please note, that the videos arrive on-time with no noticeable delay. Figure 7 shows that the actual delay is indeed negligible.

From the above results it is clear that simultaneous live videos transmission can be supported by an Emergency Response Ad hoc Network, if the nodes are equipped with multiple 
wireless interfaces and use multiple channels.

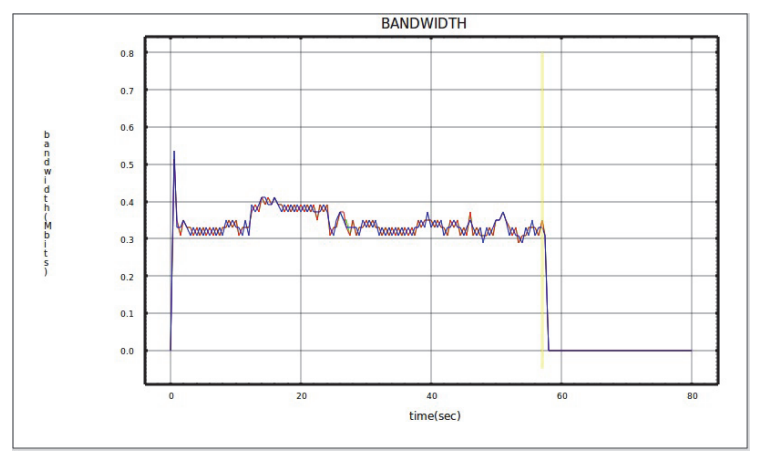

Figure 5: Bandwidth usage for 3 videos transmission with MIMC support.

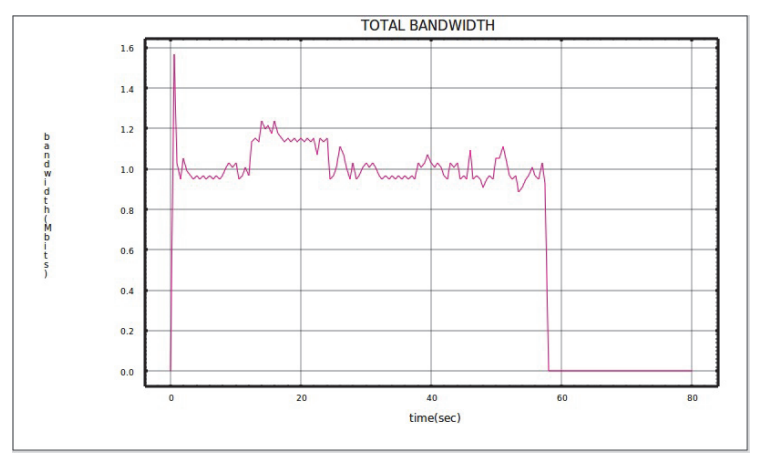

Figure 6: Cumulative bandwidth for 3 videos transmission with MIMC support.

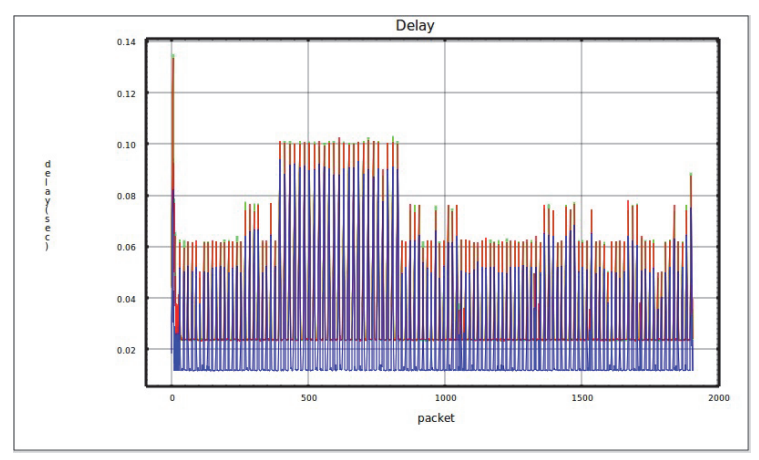

Figure 7: Delay for 3 videos transmission with MIMC support.

From the initial results it seems that two wireless interfaces per node are sufficient for the number of nodes and the number of videos expected to be transmitted within each emergency response team. However the centre node of each team acts as a sink for the videos and requires more wireless interfaces in order to be able to simultaneously receive all the videos.

Another way to overcome the difficulties of transmitting multiple videos in such an ad hoc network is to use rate adaptation and reduce the video quality when the available bandwidth is less that required in order to accommodate all videos (see e.g., Adam et al., 2012).

This is clearly not the preferred solution but may be used if the nodes are only equipped with one wireless interface.

In order to evaluate this solution we introduce rate adaptation in the video transmission. So the simulations were also run with rate adaptation enabled for all video transmissions.

Figure 8 shows the videos' transmission rates of three simultaneous video transmissions with rate adaptation. The figure is similar to Figure 2, with the three videos having rates that fluctuate a lot, but in this case the video rates adapt to the available bandwidth (by dropping a bit the video quality) and the videos finish close to their intended time.

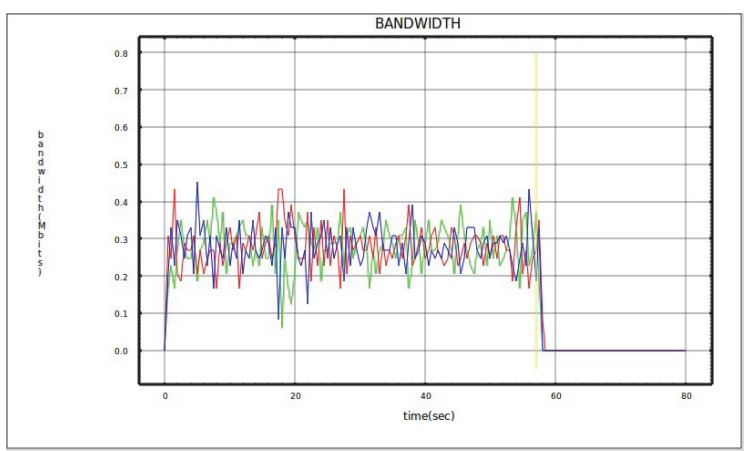

Figure 8: Bandwidth usage for 3 videos transmission with rate adaptation.

Figure 9 shows that delay of each vide frame remains relatively small (compared to the delay in Figure 4), and, more importantly, this delay does not increase with time. Contrary, as the system stabilizes, the delay remains less than half a second. This is not a big delay for the scenarios considered, and is reasonable for live video streaming.

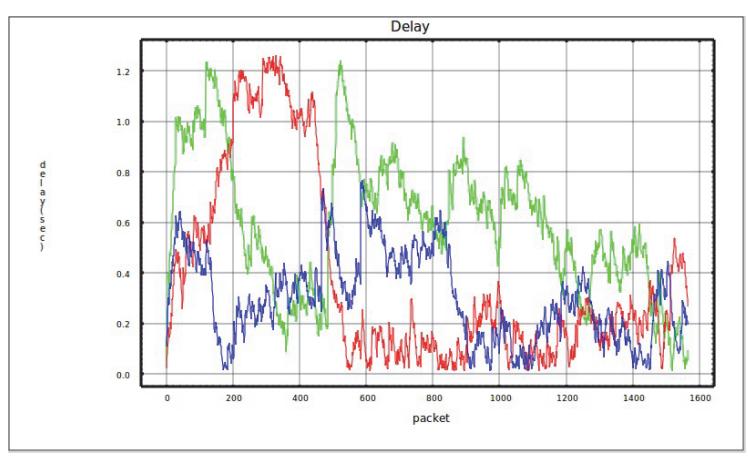

Figure 9: Delay for 3 videos transmission with rate adaptation. 
The drawback is that videos may have reduced quality during some periods. Figure 10 shows the PSNR of the transmitted videos. The PSNR stabilizes to a lower than perfect value, and this corresponds to the maximum bandwidth that is available to each video.

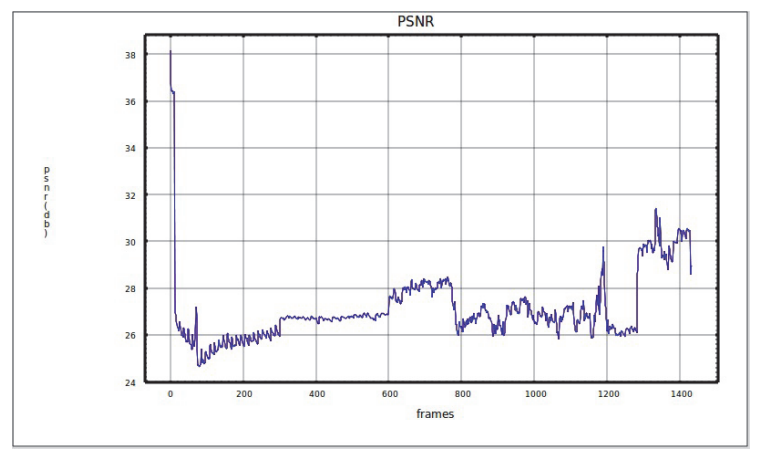

Figure 10: PSNR for 3 videos transmission with rate adaptation.

However, the received videos, judged subjectively, by human viewers, although they have noticeable lower quality, they do not seem to differ significantly from the original. In any case the received video are good enough for their intended purpose; to give the centre a clear view of what is happening in the visible field of each team member with enough clarity. So the reduction in quality does introduce any vital information loss in the scenarios considered.

Figure 11 shows a comparison of the delay for each packet transmitted in the three cases considered (green: transmission with no rate adaptation and no MIMC support, red: with MIMC support, and blue: with rate adaptation). In the case of rate adaptation, fewer packets are transmitted as the video quality is reduced.

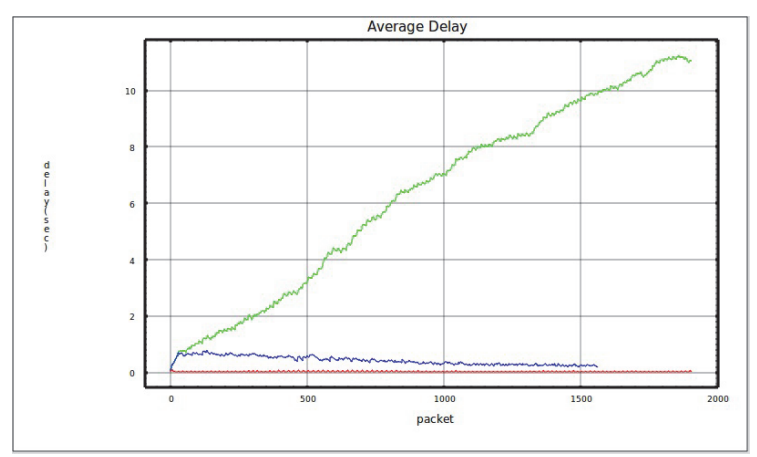

Figure 11: Comparison of the delay of each packet for the three cases considered.

The comparison demonstrates that using multiple interfaces and multiple channels should be the preferred solution for transmitting multiple live video streams in Emergency Response Ad hoc Networks. Rate adaptation should be considered only if the equipment for using multiple interfaces and multiple channels is not available. Clearly using an ad hoc network without special considerations for video transmission is, actually, not an option.

\section{CONCLUSIONS \& FUTURE WORK}

Mobile Ad hoc NETworks (MANETs) are becoming more essential to wireless communications due to growing popularity of mobile devices. MANETS are also essential in Emergency Response situation where network information flow between deployed units is vital. However, MANETs do not seem to effectively support multimedia applications and especially video transmission. In this work we evaluate the multi-interfaces and multi-channels Video Transmission in Emergency Response Ad hoc Networks. The simulation evaluation performed shows that multi-interfaces and multi-channels video transmission offers many advantages in Video Transmission in Emergency Response Ad hoc Networks. This means that the first responders should be equipped with special nodes that have multiple wireless interfaces that can transmit simultaneously in multiple channels.

Future works includes the further study multiinterfaces and multi-channels Video Transmission in Emergency Response MANETs and the proposal of the appropriate changes in the most common MANETs routing protocols in order to get benefit of multi-interfaces and multi-channels Video Transmission approach. More specifically we plan to evaluate multipath routing in such cases and investigate if and how it improves video transmission. Furthermore, we plan to research various cross-layer mechanisms that utilize, for example SNR, to improve the routing (and rerouting) and the video transmission. Our aim is to define a set of mechanisms that can be applied in order to make video transmission in Emergency Response Ad hoc Networks feasible and efficient.

\section{REFERENCES}

Adam, G., Bouras, C., Gkamas, A., Kapoulas, V., Kioumourtzis G., 2012. A Cross-Layer Design for 
Video Transmission with TFRC in MANETs. In Proceedings of the International Conference on Data Communication Networking", DCNET (2012). Rome, Italy.

Agüero Calvo, R., Pérez Campo, J., 2007. Adding Multiple Interface Support in NS-2, available at $\mathrm{http} / /$ personales.unican.es/aguerocr/files/ucMultilface sSupport.pdf

Clausen, T., Jacquet, P., 2003. Optimized Link State Routing Protocol (OLSR). RFC 3626, IETF.

Dwivedi, SK, Shukla, NK, Pandey, S., 2012. A hybrid wireless network model for post disaster relief operations. International Journal of Soft Computing and Engineering, ISSN: 2231-2307, Volume-1, Issue6, January.

Gharavi, H., 2008. Multichannel mobile ad hoc links for multimedia communications. In Proceedings of the IEEE. Vol. 96, no 1, pp. 77-96.

Goyal, P., Parmar, V., Rishi, R., 2011. MANET: Vulnerabilities, Challenges, attacks, Application, In IJCEM International Journal of Computational Engineering \& Management, Vol. 11.

Hosseini-Pozveh, M., Nematbakhsh, M. A., Movahhedinia, N., 2009. GPS-Enhanced AODV Routing. In Proceedings of the 2009 International Conference on Wireless Networks (ICWN'09). pp. 1316. Monte Carlo Resort, Las Vegas, Nevada, USA.

Jiazi, Y., 2008. A Survey on the Applications of MANET. Polytech'Nantes.

Johnson, D., Hu, Y., Maltz, D., 2007. The Dynamic Source Routing Protocol (DSR) for Mobile Ad Hoc Networks for IPv4. RFC 4728, IETF.

Karp, B., Kung, H. T., 2000. GPSR: Greedy Perimeter Stateless Routing for Wireless", In Proceedings of the 6th annual international conference on Mobile computing and networking (MobiCom '00). Boston, USA, August 6-11.

Ko, Y.-B., Vaidya, N., 2000. Location-aided routing (LAR) in mobile ad hoc networks. In Journal of Wireless Networks, Volume 6, Issue 4, 307- 32.

Kumar, N. R., Kumar, A. T., 2011. A Framework for Disaster Management using Wireless. In Proceedings of the 2011 International Conference on Communication, Computing \& Security. Rourkela, Odisha, India, February 12-14.

Panaousis, E., Ramrekha, T., Millar, G., Politis, C., 2010. Adaptive and Secure Routing Protocol for Emergency Mobile Ad Hoc Networks, In International Journal of Wireless \& Mobile Networks. 2.2, 62-78.

Perkins, C., Belding-Royer, E., Das, S., 2003. Ad hoc OnDemand Distance Vector (AODV) Routing. RFC 3561, IETF.

Raniwala, A., Chiueh T.-c., 2005. Architecture and Algorithms for an IEEE 802.11-Based Multi-Channel Wireless Mesh Network. In Proceedings of IEEE Infocom ' 05 .

Schulzrinne, H., Casner, S., Frederick, R., Jacobson, V., 2003. RTP: A Transport Protocol for Real-Time Applications, RFC 3550, IETF.
Wang, N.-C., Huang, Y.-F., Chen, J.-S. Wang, S.M., Chen, C.-L., 2009. An Improved Location-Aided Routing Protocol for Mobile Ad Hoc Networks with Greedy Approach. In WSEAS Transactions on Communications, Volume 8 Issue 8, pp. 970-979. 